\title{
一种 $\mathrm{N}, \mathrm{N}$-二甲基乙酰胺提供亚甲基在水相中构建亚甲基双酰胺的方法
}

\author{
陈 洁 $a$ 毛金成 $*, a, b$ 郑 洋 ${ }^{a}$ \\ ( ${ }^{a}$ 苏州大学材料与化学化工学部 苏州 215123) \\ $\left({ }^{b}\right.$ 西南石油大学油气藏地质与开发国家重点实验室 成都 610500)
}

\begin{abstract}
摘要 双酰胺的结构在合成仿肽类化合物中具有十分重要的作用. 以酰胺为底物, $N, N$-二甲基乙酰胺(DMAC)提供亚甲 基, $\mathrm{Na}_{2} \mathrm{~S}_{2} \mathrm{O}_{8}$ 为氧化剂, $\left.\mathrm{NH}_{4}\right)_{2} \mathrm{Ce}\left(\mathrm{NO}_{3}\right)_{6}$ 为催化剂, 在水相中反应合成亚甲基双酰胺化合物. 采用 ${ }^{1} \mathrm{H} \mathrm{NMR},{ }^{13} \mathrm{C} \mathrm{NMR}$ 和 LRMS 对目标产物进行了表征. 研究表明该方法具有环境友好, 后处理操作简单和产率高等优点, 为亚甲基双酰胺化 合物提供了新的方法.
\end{abstract}

关键词 酰胺; 亚甲基双酰胺; 水相

\section{Construction of Methylenebisamides in Aqueous Phase Using $\mathrm{N}, \mathrm{N}$-Dimethylacetamide to Provide Methylene}

\author{
Chen, Jie ${ }^{a} \quad$ Mao, Jincheng ${ }^{*, a, b} \quad$ Zheng, Yang $^{a}$ \\ ( ${ }^{a}$ College of Chemistry, Chemical Engineering and Materials Science, Soochow University, Suzhou 215123) \\ $\left({ }^{b}\right.$ State Key Laboratory of Oil and Gas Reservoir Geology and Exploitation, Southwest Petroleum University, \\ Chengdu 610500)
}

\begin{abstract}
A variety of bisamides which are key fragments in the formation of peptidomimetic compounds were synthesized. An effective method to synthesize methylenebisamides is by the reaction of amides with $N, N$-dimethylacetamide (DMAC) using $\mathrm{Na}_{2} \mathrm{~S}_{2} \mathrm{O}_{8}$ as the oxidant and $\left(\mathrm{NH}_{4}\right)_{2} \mathrm{Ce}\left(\mathrm{NO}_{3}\right)_{6}$ as the catalyst in water. The structures of product were confirmed by ${ }^{1} \mathrm{H}$ NMR, ${ }^{13} \mathrm{C}$ NMR and LRMS. The methodology will provide new strategies for the synthesis of methylenebisamides, which has the advantage of environment-friendly, simple workup and high yield.

Keywords amides; methylenebisamides; water
\end{abstract}

酰胺类化合物 $\mathbf{A}$ 大量存在于自然界中，被广泛地应 用于农业、医药行业及有机合成等重要领域. 同时, 双 酰胺结构在合成仿肽类化合物中也具有十分重要的作 用 ${ }^{[1 \sim 3]}$. 关于该类化合物的合成 ${ }^{[4]}$, 通常采用酰胺和 醛 ${ }^{[5,6]}$, 或者采用腈和甲醛 ${ }^{[7]} 反$ 应来得到. 然而, 这些合 成方法有反应时间长、产率低、反应条件苛刻、后处理 麻烦等不足. 虽然 $\mathrm{Li}$ 等 ${ }^{[8]}$ 通过酰胺和 DMSO 反应成功地 实现了亚甲基双酰胺化合物的合成, Xie 等 ${ }^{[9]}$ 报道了一 种以二甲亚砜(DMSO)为亚甲基来源生成亚甲基双酰胺 化合物的方法, 但是反应中用有机化合物作溶剂不够绿 色. 因此寻找反应过程简单、产物收率高、安全、环保、 低成本的制备方法以有效合成亚甲基双酰胺衍生物是
很有必要的。<smiles>[R][R]1ccc(C(=O)NCNC(=O)c2ccc([R])cc2)cc1</smiles>

$N$-甲基胺化合物提供亚甲基也曾被其他课题组报 道过, $\mathrm{Li}$ 等 ${ }^{[10]}$ 曾报道使用 $N, N$-二甲基苯胺(DMA) 作为亚 甲基来源生成亚甲基双(1,3-二羰)化合物(Scheme 1). 2009 年, $\mathrm{Li}$ 等 ${ }^{[11]}$ 报道了用四甲基乙二胺(TMEDA)提供 亚甲基生成亚甲基双吲哚衍生物(Scheme 1). 但是用 $\mathrm{N}, \mathrm{N}$-二甲基乙酰胺(DMAC) 来提供亚甲基仍然鲜有报

\footnotetext{
* E-mail: jcmao@suda.edu.cn
}

Received December 19, 2014; revised January 16, 2015; published online January 28, 2015.

Project supported by the Scientific Research Foundation for the Returned Overseas Chinese Scholars, State Education Ministry, the Priority Academic Program Development of Jiangsu Higher Education Institutions, and the Key Laboratory of Organic Synthesis of Jiangsu Province.

教育部留学启动基金、江苏省高校优势学科项目和江苏省有机合成重点实验室基金资助项目. 
道. 本文以 DMAC 为原料提供亚甲基和苯甲酰胺反应, 以水作为溶剂, 发展了一种简单而且绿色环保的合成亚 甲基双酰胺衍生物的方法.

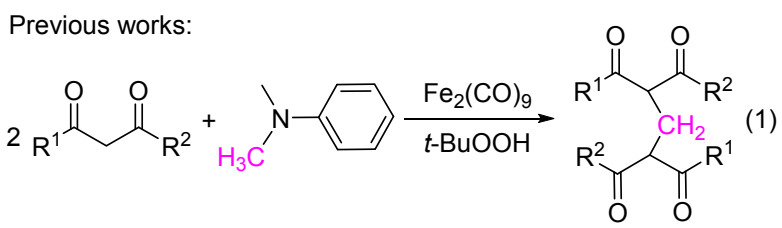

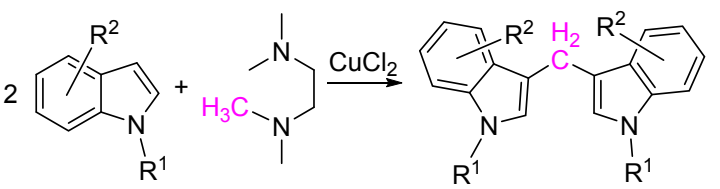

This work:<smiles>[R]c1ccc(C(=O)NCNC(=O)c2ccc([R])cc2)cc1</smiles>

图 1 亚甲基源进行的相关反应

Scheme 1 The reactions about methylene sources

\section{1 结果与讨论}

\section{1 反应条件的优化}

首先, 我们以苯甲酰胺(1a)和 DMAC (2a)反应生成 亚甲基双苯甲酰胺为典型反应，考察了催化剂、氧化剂、 反应温度和水用量等方面对 3a 产率的影响, 实验结果 如表 1 所示. 从表 1 的数据可知, 在没有催化剂的条件 下, 改变不同的氧化剂, 如 $\mathrm{Na}_{2} \mathrm{~S}_{2} \mathrm{O}_{8} 、 \mathrm{~K}_{2} \mathrm{~S}_{2} \mathrm{O}_{8}$ 、 $\left(\mathrm{NH}_{4}\right)_{2} \mathrm{~S}_{2} \mathrm{O}_{8}$, 该模型反应能得到 $42 \% \sim 52 \%$ 的收率(表 1 , Entries 1 3), 其中 $\mathrm{Na}_{2} \mathrm{~S}_{2} \mathrm{O}_{8}$ 的效果最好(表 1, Entry 1). 而当该反应在 $110{ }^{\circ} \mathrm{C}$ 的条件下进行时, 以 $\mathrm{Na}_{2} \mathrm{~S}_{2} \mathrm{O}_{8}$ 作 为氧化剂时, 反应 $24 \mathrm{~h}$ 能以 $66 \%$ 的收率得到目标化合物 3a (表 1, Entry 4). 然后, 我们又探索了不同催化剂对该 反应的影响(表 1, Entries 5 13), 由实验结果可知, 硝 酸铈铵 $\left[\left(\mathrm{NH}_{4}\right)_{2} \mathrm{Ce}\left(\mathrm{NO}_{3}\right)_{6}\right]$ 显示出了最好的催化活性. 当 改变水的用量, 将水的量从 $2 \mathrm{~mL}$ 逐渐降低到 $0.5 \mathrm{~mL}$, 产率逐渐升高再降低(表 1, Entries 13～16), 其中当水的 量为 $1 \mathrm{~mL}$ 时产率最高, 达到 $86 \%$ (表 1, Entry 15). 而当 温度升高到 $120{ }^{\circ} \mathrm{C}$ 和 $140{ }^{\circ} \mathrm{C}$, 产率分别只得到了 $76 \%$ 和 $67 \%$ (表 1, Entries 17,18).

\section{$1.21 \mathrm{a}$ 和其他 $\mathrm{N}$-甲基胺的反应}

在最优的反应条件下，我们尝试了其他 $N$-甲基胺 的反应, 实验结果如表 2 所示. 当使用 $N, N$-二甲基甲酰 胺(DMF)、 $N$-甲基乙酰胺和苯甲酰胺在最优条件下反应，
表 1 反应条件的优化 ${ }^{a}$

Table 1 Optimization of reaction conditions

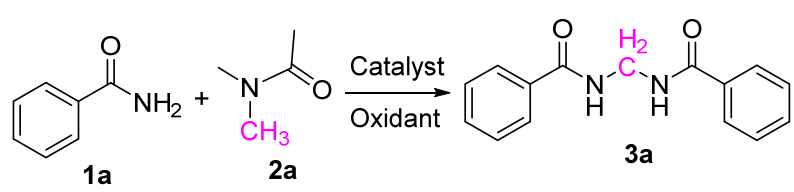

\begin{tabular}{cllcc}
\hline Entry & \multicolumn{1}{c}{ Catalyst } & \multicolumn{1}{c}{ Oxidant } & Temp. $/^{\circ} \mathrm{C}$ & Yield $^{b} / \%$ \\
\hline 1 & - & $\mathrm{Na}_{2} \mathrm{~S}_{2} \mathrm{O}_{8}$ & 100 & 52 \\
2 & - & $\mathrm{K}_{2} \mathrm{~S}_{2} \mathrm{O}_{8}$ & 100 & 48 \\
3 & - & $\left(\mathrm{NH}_{4}\right)_{2} \mathrm{~S}_{2} \mathrm{O}_{8}$ & 100 & 42 \\
4 & - & $\mathrm{Na}_{2} \mathrm{~S}_{2} \mathrm{O}_{8}$ & 110 & 66 \\
5 & $\mathrm{Cu}(\mathrm{OAc})_{2}$ & $\mathrm{Na}_{2} \mathrm{~S}_{2} \mathrm{O}_{8}$ & 110 & 53 \\
6 & $\mathrm{CuBr}$ & $\mathrm{Na}_{2} \mathrm{~S}_{2} \mathrm{O}_{8}$ & 110 & 52 \\
7 & $\mathrm{Cu}$ & $\mathrm{Na}_{2} \mathrm{~S}_{2} \mathrm{O}_{8}$ & 110 & 74 \\
8 & $\mathrm{CuI}$ & $\mathrm{Na}_{2} \mathrm{~S}_{2} \mathrm{O}_{8}$ & 110 & 68 \\
9 & $\mathrm{FeCl}$ & $\mathrm{Na}_{2} \mathrm{~S}_{2} \mathrm{O}_{8}$ & 110 & 34 \\
10 & $\mathrm{Ni}$ & $\mathrm{Na}_{2} \mathrm{~S}_{2} \mathrm{O}_{8}$ & 110 & 72 \\
11 & $\mathrm{AgOAc}$ & $\mathrm{Na}_{2} \mathrm{~S}_{2} \mathrm{O}_{8}$ & 110 & 78 \\
12 & $\mathrm{Ce}\left(\mathrm{NO}_{3}\right)_{3} 6 \mathrm{H}_{2} \mathrm{O}$ & $\mathrm{Na}_{2} \mathrm{~S}_{2} \mathrm{O}_{8}$ & 110 & 78 \\
13 & $\left(\mathrm{NH}_{4}\right)_{2} \mathrm{Ce}\left(\mathrm{NO}_{3}\right)_{6}$ & $\mathrm{Na}_{2} \mathrm{~S}_{2} \mathrm{O}_{8}$ & 110 & 80 \\
$14^{c}$ & $\left(\mathrm{NH}_{4}\right)_{2} \mathrm{Ce}\left(\mathrm{NO}_{3}\right)_{6}$ & $\mathrm{Na}_{2} \mathrm{~S}_{2} \mathrm{O}_{8}$ & 110 & 81 \\
$15^{d}$ & $\left(\mathrm{NH}_{4}\right)_{2} \mathrm{Ce}\left(\mathrm{NO}_{3}\right)_{6}$ & $\mathrm{Na}_{2} \mathrm{~S}_{2} \mathrm{O}_{8}$ & 110 & 86 \\
$16^{e}$ & $\left(\mathrm{NH}_{4}\right)_{2} \mathrm{Ce}\left(\mathrm{NO}_{3}\right)_{6}$ & $\mathrm{Na}_{2} \mathrm{~S}_{2} \mathrm{O}_{8}$ & 110 & 74 \\
17 & $\left(\mathrm{NH}_{4}\right)_{2} \mathrm{Ce}\left(\mathrm{NO}_{3}\right)_{6}$ & $\mathrm{Na}_{2} \mathrm{~S}_{2} \mathrm{O}_{8}$ & 120 & 76 \\
18 & $\left(\mathrm{NH}_{4}\right)_{2} \mathrm{Ce}\left(\mathrm{NO}_{3}\right)_{6}$ & $\mathrm{Na}_{2} \mathrm{~S}_{2} \mathrm{O}_{8}$ & 140 & 67 \\
\hline
\end{tabular}

${ }^{a}$ Reaction conditions: 1a $(0.5 \mathrm{mmol}), \mathbf{2 a}$ (4.0 equiv.), oxidant (4.0 equiv.), catalyst (5 mol\%), $\mathrm{H}_{2} \mathrm{O}(2 \mathrm{~mL}), 24 \mathrm{~h} ;{ }^{b}$ Isolated yield; ${ }^{c} \mathrm{H}_{2} \mathrm{O}(1.5 \mathrm{~mL})$; ${ }^{d} \mathrm{H}_{2} \mathrm{O}(1 \mathrm{~mL}) ;{ }^{e} \mathrm{H}_{2} \mathrm{O}(0.5 \mathrm{~mL})$.

能够分别得到 54\%和 $67 \%$ 的产率(表 2, Entries 1,3). 而 当使用 DMA，TMEDA 和苯甲酰胺反应，即使在最优条 件下也没有得到 3a(表 2, Entries 2, 4). 由上面实验可知, 该反应中 DMAC 是最好的亚甲基来源试剂.

\section{3 底物的拓展}

在最优化的反应条件下，我们考察了不同取代基团 的苯甲酰胺和 DMAC 的反应, 结果见表 3. 由表 3 可知, 苯甲酰胺以及苯环上连有中性基团的底物, 如对甲基苯 甲酰胺、对异丙基苯甲酰胺和对叔丁基苯甲酰胺与 DMAC 反应能得到 $67 \% \sim 86 \%$ 的产率(表 3, Entries 1 4). 同时，对甲氧基苯甲酰胺在最优化的条件下进行反 应也能得到 79\%的产率(表 3, Entry 5). 然而, 当苯环上 连有卤素如氯和氟, 仅能得到 53\%和 43\% 的收率(表 3, Entries 6,7).

\section{4 可能的反应机理}

基于文献报道 ${ }^{[12]}$ 和本文实验结果，推测苯甲酰胺 和 DMAC 反应生成亚甲基双酰胺化合物可能的反应机 理见 Scheme 2. DMAC 在 $\mathrm{Na}_{2} \mathrm{~S}_{2} \mathrm{O}_{8}$ 和 $\left(\mathrm{NH}_{4}\right)_{2} \mathrm{Ce}\left(\mathrm{NO}_{3}\right)_{6}$ 存 在下生成中间体 $\mathbf{A}^{[13]}$, 苯甲酰胺和中间体 $\mathbf{A}$ 亲核加成生 成化合物 $\mathbf{B}$, 化合物 $\mathbf{B}$ 在苯甲酰胺的存在下进行亲核取 代而生成亚甲基双酰胺化合物. 
表 $21 \mathbf{a}$ 和其他 $N$-甲基胺的反应 ${ }^{a}$

Table 2 Reactions of $1 \mathrm{a}$ with other $N$-methyl amines

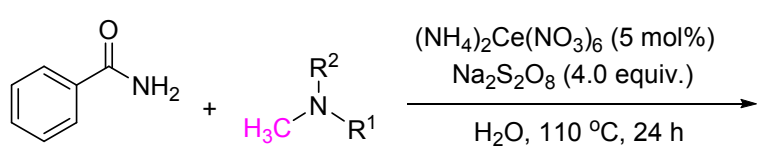

$1 a$

2<smiles>O=C(NCNC(=O)c1ccccc1)c1ccccc1</smiles>

3a

\begin{tabular}{|c|c|c|}
\hline Entry & 2 & Yield $^{b} / \%$ \\
\hline 1 & & 54 \\
\hline 2 & & ND \\
\hline 3 & $\mathrm{H}$ & 67 \\
\hline 4 & & ND \\
\hline
\end{tabular}
$\left(\mathrm{NH}_{4}\right)_{2} \mathrm{Ce}\left(\mathrm{NO}_{3}\right)_{6}(5 \mathrm{~mol} \%), \mathrm{H}_{2} \mathrm{O}(1 \mathrm{~mL}), 24 \mathrm{~h} ;{ }^{b}$ Isolated yield.<smiles>CC(=O)N(C)CCS(=O)(=O)O[Na]</smiles><smiles>CC(=O)N(C)CNC(=O)c1ccccc1</smiles><smiles>O=C(NCNC(=O)c1ccccc1)c1ccccc1</smiles>

图 2 可能的反应机理

Scheme 2 The possible mechanism

\section{2 结论}

以 $\left(\mathrm{NH}_{4}\right)_{2} \mathrm{Ce}\left(\mathrm{NO}_{3}\right)_{6} / \mathrm{Na}_{2} \mathrm{~S}_{2} \mathrm{O}_{8}$ 体系作为反应促进体系, 在 $110{ }^{\circ} \mathrm{C}$ 和水作为溶剂的条件下, 通过苯甲酰胺类化 合物和 DMAC 反应, 高收率地实现了一系列亚甲基双 酰胺化合物的合成. 此方法具有反应原料易得、后处理 操作简单和产品纯度高等优点, 特别是在水中进行有机 化合物的反应，符合绿色化学的发展方向.
表 3 底物的拓展 ${ }^{a}$

Table 3 Scope of the substrates<smiles>[R]c1ccc(C(N)=O)cc1</smiles><smiles>[R][R]1ccc(C(=O)NCNC(=O)c2cc[R]([R])cc2)cc1</smiles>

3

Entry $\quad$ Product $\quad$ Yield $^{b} \%$

2<smiles>O=C(NCNC(=O)c1ccccc1)c1ccccc1</smiles>

1 3a 3b

3<smiles>Cc1ccc(C(=O)NCNC(=O)c2ccc(C)cc2)cc1</smiles>

$3 c$<smiles>CC(C)c1ccc(C(=O)NCNC(=O)c2ccc(C(C)C)cc2)cc1</smiles>
75 4<smiles>CC(C)(C)c1ccc(C(=O)NCNC(=O)c2ccc(C(C)(C)C)cc2)cc1</smiles>

5<smiles>COc1ccc(C(=O)NCNC(=O)c2ccc(OC)cc2)cc1</smiles>

6<smiles>O=C(NCNC(=O)c1ccc(Cl)cc1)c1ccc(Cl)cc1</smiles>

7<smiles>O=C(NCNC(=O)c1ccc(F)cc1)c1ccc(F)cc1</smiles>

${ }^{a}$ Reaction conditions: 1 ( 0.5 mmol), 2 (4.0 equiv.), $\mathrm{Na}_{2} \mathrm{~S}_{2} \mathrm{O}_{8}$ (4.0 equiv.), $\left(\mathrm{NH}_{4}\right)_{2} \mathrm{Ce}\left(\mathrm{NO}_{3}\right)_{6}(5 \mathrm{~mol} \%), \mathrm{H}_{2} \mathrm{O}(1.0 \mathrm{~mL}), 24 \mathrm{~h} ;{ }^{b}$ Isolated yield.

\section{3 实验部分}

\section{1 仪器与试剂}

熔点测定采用 XT-4 型双目显微熔点测定仪; 核磁 用 Bruker 公司 $400 \mathrm{MHz}$ 核磁共振仪, TMS 为内标; 质谱 
用 Agilent 气质联用仪测定. 苯甲酰胺衍生物的合成按 照 Chill ${ }^{[13]}$ 的报道.

\section{2 实验方法}

以化合物 $\mathbf{1 a}$ 的合成为例. 在 $10 \mathrm{~mL}$ 的耐压试管中 加入 $0.5 \mathrm{mmol}$ 苯甲酰胺, $2.0 \mathrm{mmol}$ 过硫酸钠, 0.025 $\mathrm{mmol}$ 硝酸铈铵, $1.0 \mathrm{~mL}$ 水、2.0 mmol DMAC, 在 $110{ }^{\circ} \mathrm{C}$ 的条件下搅拌反应 $24 \mathrm{~h}$. 反应结束后, 将反应体系冷却 至室温, 将所得反应液经柱层析纯化得到产物 $\mathbf{3 a}$.

$N, N$-亚甲基双苯甲酰胺(3a): 白色固体，产率 $86 \%$. m.p. $215 \sim 217{ }^{\circ} \mathrm{C}$ (文献值 ${ }^{[14]}: 215 \sim 217{ }^{\circ} \mathrm{C}$ ); ${ }^{1} \mathrm{H}$ NMR (400 MHz, DMSO-d $\left.)_{6}\right) \delta: 9.06(\mathrm{t}, J=5.4 \mathrm{~Hz}, 2 \mathrm{H}), 7.93 \sim$ $7.91(\mathrm{~m}, 4 \mathrm{H}), 7.55 \sim 7.44(\mathrm{~m}, 6 \mathrm{H}), 4.89$ (t, $J=5.6 \mathrm{~Hz}, 2 \mathrm{H})$; ${ }^{13} \mathrm{C}$ NMR (100 MHz, DMSO- $\left.d_{6}\right) \delta: 167.0,134.4,131.9$, 128.7, 127.9, 45.7; LRMS m/z: $255.1(\mathrm{M}+\mathrm{H})^{+}$.

$N, N$-亚甲基双(4-甲基苯甲酰胺)(3b): 白色固体，产 率 67\%. m.p. 216 217 ${ }^{\circ} \mathrm{C}$ (文献值 ${ }^{[15]}$ : 216 218 ${ }^{\circ} \mathrm{C}$ ); ${ }^{1} \mathrm{H}$ NMR (400 MHz, DMSO- $\left.d_{6}\right) \delta: 8.99(\mathrm{t}, J=5.4 \mathrm{~Hz}, 2 \mathrm{H})$, $7.83(\mathrm{~d}, J=8.0 \mathrm{~Hz}, 4 \mathrm{H}), 7.26$ (d, $J=8.0 \mathrm{~Hz}, 4 \mathrm{H}), 4.86(\mathrm{t}$, $J=5.6 \mathrm{~Hz}, 2 \mathrm{H}), 2.34(\mathrm{~s}, 6 \mathrm{H}) ;{ }^{13} \mathrm{C} \mathrm{NMR}(100 \mathrm{MHz}$, DMSO- $\left.d_{6}\right) \delta: 166.8,141.8,131.6,129.3,127.9,45.6,21.4$; LRMS $m / z: 283.1(\mathrm{M}+\mathrm{H})^{+}$.

$N, N$-亚甲基双(4-异丙基苯甲酰胺)(3c)：白色固体， 产率 $83 \%$. m.p. $188 \sim 189{ }^{\circ} \mathrm{C}$; ${ }^{1} \mathrm{H}$ NMR $(400 \mathrm{MHz}$, DMSO- $\left.d_{6}\right) \delta: 8.98(\mathrm{t}, J=5.6 \mathrm{~Hz}, 2 \mathrm{H}), 7.86(\mathrm{~d}, J=8.4 \mathrm{~Hz}$, $4 \mathrm{H}), 7.31(\mathrm{~d}, J=8.4 \mathrm{~Hz}, 4 \mathrm{H}), 4.88(\mathrm{t}, J=5.6 \mathrm{~Hz}, 2 \mathrm{H})$, $2.95 \sim 2.88(\mathrm{~m}, 2 \mathrm{H}), 1.20(\mathrm{~d}, J=6.8 \mathrm{~Hz}, 12 \mathrm{H}) ;{ }^{13} \mathrm{C} \mathrm{NMR}$ $\left(100 \mathrm{MHz}, \mathrm{DMSO}-d_{6}\right) \delta: 166.9,152.4,132.1,128.0,126.6$, 45.5, 33.8, 24.1; HRMS calcd for $\mathrm{C}_{21} \mathrm{H}_{26} \mathrm{~N}_{2} \mathrm{O}_{2}(\mathrm{M}+\mathrm{H})^{+}$: 339.2073, found 339.2066.

$N, N$-亚甲基双(4-叔丁基苯甲酰胺)(3d)：白色固体， 产率 75\%. m.p. 259 260 ${ }^{\circ} \mathrm{C}$; ${ }^{1} \mathrm{H}$ NMR (400 MHz, DMSO- $\left.d_{6}\right) \delta: 8.96(\mathrm{t}, J=5.6 \mathrm{~Hz}, 2 \mathrm{H}), 7.85(\mathrm{~d}, J=8.5 \mathrm{~Hz}$, 4H), 7.47 (d, $J=8.4 \mathrm{~Hz}, 4 \mathrm{H}), 4.87$ (t, $J=5.6 \mathrm{~Hz}, 2 \mathrm{H}), 1.29$ $(\mathrm{s}, 18 \mathrm{H}) ;{ }^{13} \mathrm{C} \mathrm{NMR}\left(100 \mathrm{MHz}, \mathrm{DMSO}-d_{6}\right) \delta: 166.8,154.6$, 131.7, 127.8, 125.5, 45.5, 35.1, 31.4; HRMS calcd for $\mathrm{C}_{23} \mathrm{H}_{30} \mathrm{~N}_{2} \mathrm{O}_{2}(\mathrm{M}+\mathrm{H})^{+}: 367.2386$, found 367.2389.

$N, N$-亚甲基双(4-甲氧基苯甲酰胺)(3e): 白色固体, 产率 79\%. m.p. 206 $208{ }^{\circ} \mathrm{C}$ (文献值 ${ }^{[15]}$ : 207.5 209 ${ }^{\circ} \mathrm{C}$ ); ${ }^{1} \mathrm{H}$ NMR (400 MHz, DMSO- $\left.d_{6}\right) \delta: 8.90$ (t, $\left.J=5.6 \mathrm{~Hz}, 2 \mathrm{H}\right)$, 7.91 (d, $J=8.8 \mathrm{~Hz}, 4 \mathrm{H}), 6.99$ (d, $J=8.8 \mathrm{~Hz}, 4 \mathrm{H}), 4.86$ (t,
$J=5.6 \mathrm{~Hz}, 2 \mathrm{H}), 3.80(\mathrm{~s}, 6 \mathrm{H}) ;{ }^{13} \mathrm{C} \mathrm{NMR}(100 \mathrm{MHz}$, DMSO- $\left.d_{6}\right) \delta: 166.4,162.2,129.8,126.7,113.9,55.8,45.6$; LRMS $m / z: 315.1(\mathrm{M}+\mathrm{H})^{+}$.

$N, N$-亚甲基双(4-氯苯甲酰胺)(3f)：白色固体，产率 53\%. m.p. 238 240 ${ }^{\circ} \mathrm{C}$ (文献值 ${ }^{[15]}: 239 \sim 241{ }^{\circ} \mathrm{C}$ ); ${ }^{1} \mathrm{H}$ NMR (400 MHz, DMSO- $\left.d_{6}\right) \delta: 9.18(\mathrm{t}, J=5.4 \mathrm{~Hz}, 2 \mathrm{H})$, $7.93(\mathrm{~d}, J=8.4 \mathrm{~Hz}, 4 \mathrm{H}), 7.54$ (d, $J=8.4 \mathrm{~Hz}, 4 \mathrm{H}), 4.86$ (t, $J=5.6 \mathrm{~Hz}, 2 \mathrm{H}) ;{ }^{13} \mathrm{C} \mathrm{NMR}\left(100 \mathrm{MHz}, \mathrm{DMSO}-d_{6}\right) \delta: 166.0$, 136.7, 133.1, 129.9, 128.8, 45.7; LRMS m/z: $323.0(\mathrm{M}+$ $\mathrm{H})^{+}$.

$N, N$-亚甲基双(4-氟苯甲酰胺) (3g): 白色固体，产 率 43\%. m.p. 234 235 ${ }^{\circ} \mathrm{C}$; ${ }^{1} \mathrm{H}$ NMR (400 MHz, DMSO$\left.d_{6}\right) \delta: 9.11(\mathrm{~s}, 2 \mathrm{H}), 7.99(\mathrm{~m}, 4 \mathrm{H}), 7.29(\mathrm{t}, J=8.8 \mathrm{~Hz}, 4 \mathrm{H})$, $4.86(\mathrm{t}, J=5.2 \mathrm{~Hz}, 2 \mathrm{H}) ;{ }^{13} \mathrm{C} \mathrm{NMR}\left(100 \mathrm{MHz}, \mathrm{DMSO}-d_{6}\right)$ $\delta$ : $165.9,164.5(\mathrm{~d}, J=248.0 \mathrm{~Hz}), 130.9(\mathrm{~d}, J=2.0 \mathrm{~Hz})$, $130.6(\mathrm{~d}, J=9.0 \mathrm{~Hz}), 115.7$ (d, $J=21.0 \mathrm{~Hz}), 45.6$; HRMS calcd for $\mathrm{C}_{15} \mathrm{H}_{12} \mathrm{~F}_{2} \mathrm{~N}_{2} \mathrm{O}_{2}(\mathrm{M}+\mathrm{H})^{+}$: 290.0945, found 290.0951 .

\section{References}

[1] Alemán, C.; Puiggali, J. J. Org. Chem. 1995, 60, 910.

[2] Pallai, P. V.; Struthers, R. S.; Goodman, M.; Moroder, L.; Wunsch, E.; Vale, W. Biochemistry. 1985, 24, 1933.

[3] Rodriguez, M.; Dubreuil, P.; Bali, J.-P.; Martinez, J. J. Med. Chem. 1987, 30, 758 .

[4] Kamogawa, H.; Minoura, S.; Miyama, S. J. Polym. Sci., Polym. Chem. 1990, 28, 1435.

[5] Fernández, A. H.; Alvarez, R. M.; Abajo, T. M. Synthesis 1996, 1299.

[6] Gilbert, E. E. Synthesis 1972, 30.

[7] Magat, E. E.; Faris, B. F.; Reith, J. E.; Salisbury, L. F. J. Am. Chem. Soc. 1951, 73, 1028.

[8] Wang, Q.; Sun, L.; Jiang, Y.; Li, C. Beilstein J. Org. Chem. 2008, 4, 51.

[9] Pan, L.; Huang, L.; Xie, C. Lett. Org. Chem. 2013, 10, 770.

[10] Zhang, L.; Peng, C. Zhao, D.; Wang, Y.; Fu, H.-J.; Shen, Q.; Li, J.-X. Chem. Commun. 2012, 48, 5928.

[11] Li, H.; He, Z.; Guo, X.; Li, W.; Zhao, X.; Li, Z. Org. Lett. 2009, 11, 4176.

[12] Li, Y.; Xue, D.; Lu, W.; Wang, C.; Liu, Z.-T.; Xiao, J. Org. Lett. 2014, 16,66

[13] Chill, S. T.; Mebane, R. C. Synth. Commun. 2010, 40, 2014

[14] Ohashi, Y.; Terao, Y.; Sekiya, M. Chem. Pharm. Bull. 1978, 26, 653.

[15] Tanimoto, S.; Horikawa, J.; Oda, R. J. Synth. Org. Chem., Jpn. $1969,27,59$. 